\title{
Regulation of neuraminidase expression in Streptococcus pneumoniae
}

\author{
Luciana Gualdi ${ }^{1}$, Jasvinder Kaur Hayre ${ }^{1}$, Alice Gerlini ${ }^{1}$, Alessandro Bidossi', Leonarda Colomba ${ }^{1}$, Claudia Trappetti ${ }^{1,5}$, \\ Gianni Pozzi ${ }^{1}$, Jean-Denis Docquier ${ }^{1}$, Peter Andrew ${ }^{2}$, Susanna Ricci ${ }^{1}$ and Marco R Oggioni ${ }^{1,3,4,5^{*}}$
}

\begin{abstract}
Background: Sialic acid (N-acetylneuraminic acid; NeuNAc) is one of the most important carbohydrates for Streptococcus pneumoniae due of its role as a carbon and energy source, receptor for adhesion and invasion and molecular signal for promotion of biofilm formation, nasopharyngeal carriage and invasion of the lung.

Results: In this work, NeuNAc and its metabolic derivative N-acetyl mannosamine (ManNAc) were used to analyze regulatory mechanisms of the neuraminidase locus expression. Genomic and metabolic comparison to Streptococcus mitis, Streptococcus oralis, Streptococcus gordonii and Streptococcus sanguinis elucidates the metabolic association of the two amino sugars to different parts of the locus coding for the two main pneumococcal neuraminidases and confirms the substrate specificity of the respective $A B C$ transporters. Quantitative gene expression analysis shows repression of the locus by glucose and induction of all predicted transcriptional units by ManNAc and NeuNAc, each inducing with higher efficiency the operon encoding for the transporter with higher specificity for the respective amino sugar. Cytofluorimetric analysis demonstrated enhanced surface exposure of NanA on pneumococci grown in NeuNAc and ManNAc and an activity assay allowed to quantify approximately twelve times as much neuraminidase activity on induced cells as opposed to glucose grown cells.
\end{abstract}

Conclusions: The present data increase the understanding of metabolic regulation of the nanAB locus and indicate that experiments aimed at the elucidation of the relevance of neuraminidases in pneumococcal virulence should possibly not be carried out on bacteria grown in glucose containing media.

Keywords: Sialic acid, Metabolic regulation, Carbon catabolite repression

\section{Background}

Streptococcus pneumoniae is a common inhabitant of the upper respiratory tract and it is also a major human pathogen. The self-limited carriage episodes represent the most common interaction between pneumococci and the host. However, in some cases, such asymptomatic interaction can progress to invasive disease [1]. Of the many factors influencing the interaction of the bacterium with the host, numerous extracellular glycosylhydrolases and carbohydrate transporters have been found to play significant roles [2]. The sialidases or neuraminidases, which are able to cleave terminal sialic acid (neuraminic acid, NeuNAc) residues present in O-linked and $\mathrm{N}$-linked glycans, have since long received special

\footnotetext{
* Correspondence: oggioni@unisi.it

${ }^{1}$ Dipartimento di Biotecnologie, Università di Siena, Siena, Italy

${ }^{3}$ UOC Batteriologia, Azienda Universitaria Ospedaliera Senese, Siena, Italy

Full list of author information is available at the end of the article
}

attention as virulence determinants [3,4]. Direct interaction of the microbial sialidases with host glycoproteins resulting in exposure of additional attachment sites on host cells was the mechanisms most frequently found to be involved in virulence [5-7]. Recently such interaction was found to be directly involved in invasion $[8,9]$. Despite the impact of sialidases in pneumococcal pathogenesis, metabolic implications have received less attention, including the utilisation of sialic acid as a carbon source on the glucose-free mucosal surfaces [10-16]. Sialic acid has recently been described by us and others to act as a molecular signal for pneumococci, resulting in increased carriage and translocation of bacteria to the lung $[10,14,17]$.

Given the prominent role of sialidases in hostpathogen interaction, it is not surprising that pneumococci harbour three sialidases, two of which, NanA and $\mathrm{NanB}$, are common to all pneumococci and the third,

\section{Biomed Central}

(c) 2012 Gualdi et al.; licensee BioMed Central Ltd. This is an Open Access article distributed under the terms of the Creative Commons Attribution License (http://creativecommons.org/licenses/by/2.0), which permits unrestricted use, distribution, and reproduction in any medium, provided the original work is properly cited. 
$\mathrm{NanC}$, is present in only $51 \%$ of strains [18]. Structural and functional analysis of the three enzymes indicated possible different roles. NanA is a first-line virulence factor for sialic acid removal, the trans-sialidase NanB is involved in the metabolic use of sialic acid, and NanC has a regulatory role, being able to produce and remove an intermediate metabolic compound which also acts as sialidase inhibitor $[19,20]$. The conserved nan $A B$ locus that comprises the genes between SPG1583 and SPG1601 in strain G54 (SP1674-94 in TIGR4) was identified as the cluster responsible for uptake and metabolism of sialic acid [16,21-23]. In addition to the extracellular sialidases NanA and NanB, the regulon encodes two $A B C$ transporters, one of which responsible for sialic acid and $\mathrm{N}$-acetyl mannosamine uptake SPG1589-91 (satABC) and the other (SP1596-8) for uptake of $\mathrm{N}$-acetyl mannosamine alone [14,23]. In addition to the $\mathrm{ABC}$ transporters the locus encodes a PTS uptake system for glucosamine, and the remaining genes encode for enzymes involved in sialic acid metabolism [23]. In vitro this operon was found to be the main cluster showing differential expression in pneumococcal opacity variants and was predicted to be composed of four predicted transcriptional units [21]. During infection, the $n a n A B$ operon was found to be upregulated in pneumonia and meningitis compared to growth in blood $[24,25]$. Much less information is available on the nanC operon, except for the analysis of the enzymatic function of the sialidase NanC [20] and its recent implication as an alternative system for the uptake of sialic acid [23].

The present work aims at performing a functional analysis of the operon in order to gain further insight into the metabolic regulation of this locus.

\section{Results}

The NanAB locus conservation in oral streptococci

As a first approach to elucidate the metabolic relevance and regulation of the different predicted transcriptional units of the $n a n A B$ regulon, we performed a genomic comparison amongst related streptococcal species, including pneumococcal strain G54, S. mitis B6, S. oralis Uo5, S. sanguis SK36 and S. gordonii V288 (Figure 1A and Table 1). With respect to S. pneumoniae G54, S. mitis B6 and $S$. oralis Uo5, these showed an identical organization for part of the locus including the neuraminidase A (nanA), the orthologs of the sat $A B C$ transporter SPG1589-91 and the genomic regions encoding the transcriptional regulator and orthologues of the enzymes involved in the first steps of sialic acid metabolism, i.e. $\mathrm{N}$-acetylneuraminate lyase and $\mathrm{N}$-acetylmannosamine kinase (Figure 1). In contrast to pneumococci these two species, S. mitis and S. oralis, did not possess the sialidase NanB, the second ABC transporter SPG1596-8, and the PTS system. In contrast to $S$. mitis and S. oralis, $S$. gordonii V288 and S. sanguinis SK36 did not possess any neuraminidases. Interestingly both S. gordonii and S. sanguis still possess orthologs of the $\mathrm{N}$-acetylneuraminate lyase, $\mathrm{N}$-acetylmannosamine kinase and $\mathrm{N}$-acetylmannosamine-6-phosphate 2-epimerase predicted to be necessary for metabolism of sialic acid (Figure 1A,B; Table 1). In addition, S. gordonii and $S$. sanguis possessed the transcriptional regulator and the orthologs of the pneumococcal SPG1596-8 ABC transporter. In contrast to S. pneumoniae, S. gordonii and S. sanguis possess neither the PTS system nor the SPG1589-91 satABC transporter. To check the amino sugar metabolism of these three different species of streptococci growth curves and fermentation assay on NeuNAc and ManNAc were performed. The growth curves show that $S$. gordonii grows only in presence of ManNAc, while S. mitis and S. pneumoniae are capable of growth on both amino sugars (Figure 2A,C). Similarly in the fermentation assay only $S$. gordonii acidified efficiently the medium in presence of ManNAc, while both S. pneumoniae and S. mitis metabolised efficiently only NeuNAc, with some acidification of the medium with ManNAc by the pneumococcus (Figure 2D).

\section{Neuraminidase locus induction in S. pneumoniae}

The putative regulator of the nanAB locus SPG1583 contains a classical N-terminal helix-turn-helix motif and a SIS domain, found in many phosphosugar binding proteins including transcriptional regulators binding to the phosphorylated end-products of the pathways [26]. Given the probable catabolic pathway of sialic acid (Figure 1B), ManNAc-6-phosphate appears to be the most probably compound having a regulatory role on the expression of pneumococcal neuraminidase operon and thus possibly in sialic acid metabolism [23]. Therefore we analysed the growth curves and the expression levels of some key genes associated with the transporter systems in the neuraminidase locus. First we compared the growth in the presence of ManNAc as a carbon source of a un-encapsulated G54 derivative FP65 and two isogenic mutants devoid of the whole nan $A B$ locus and of the transcriptional regulator SPG1583 respectively (Figure $3 \mathrm{~A}$ ). The growth curves showed absence of growth in the presence of ManNAc for both mutants, indicating that the nan $A B$ locus is essential for efficient growth of ManNAc and that the phosphosugar binding regulator SPG1583 gene appears to acts as a transcriptional activator. Then we focused our attention on growth of the wild type strain in the presence or absence of ManNAc, preferred by us for the indication assays over NeuNAc, as this amino sugar does not acidify the medium. In these experiments bacteria initially grew on residual yeast-extract derived dextran of nonsupplemented CAT medium (40 min) and continued to 


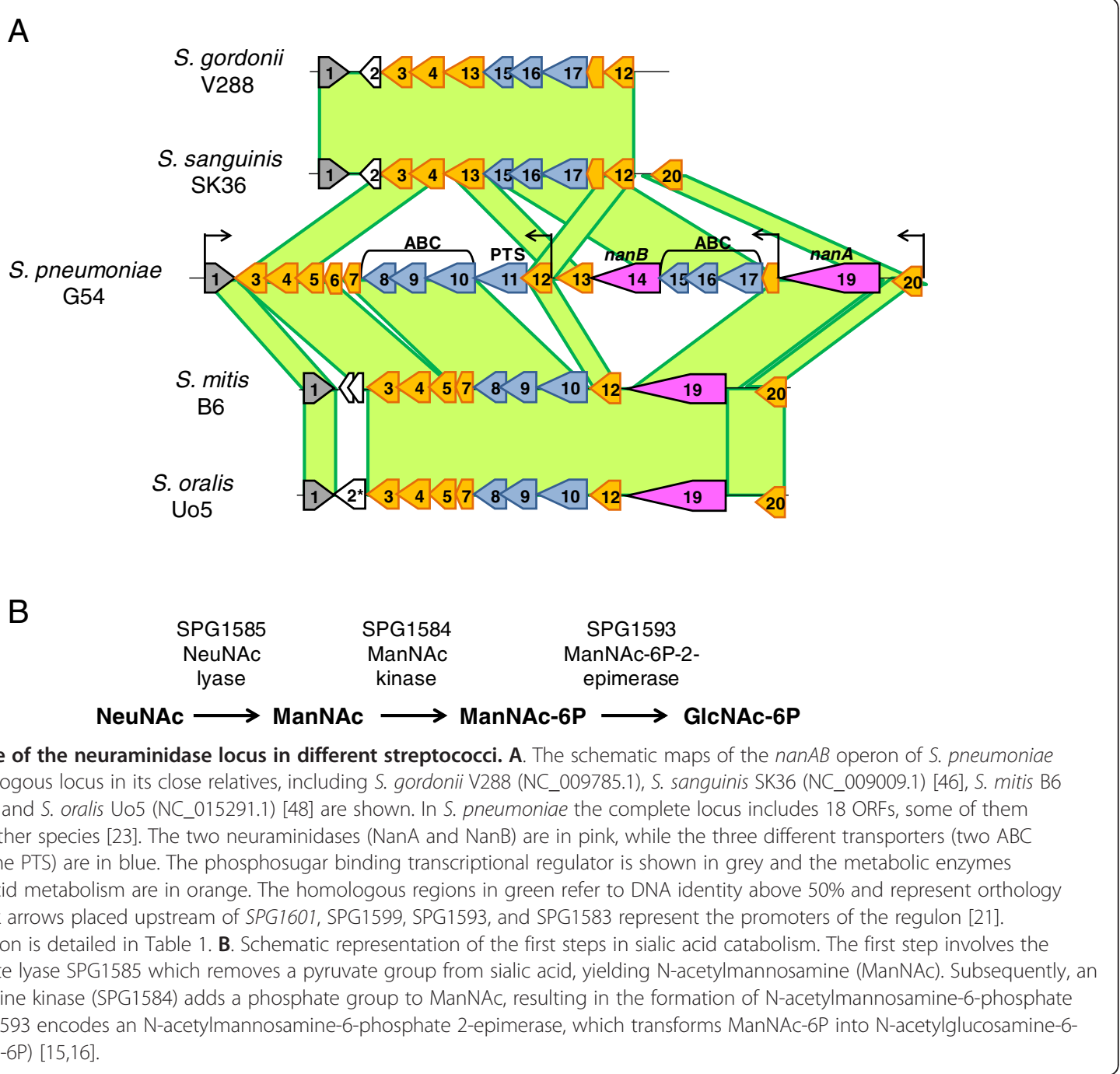

grow thereafter with a lower generation time of $140 \mathrm{~min}$ on ManNAc only (Figure 3B). For gene expression profiling bacteria were sampled in early exponential growth $\left(\mathrm{OD}_{590}=0.02\right)$, when growth was still due to the residual yeast extract-derived sugar (Figure 3B, black arrows). For bacteria grown on yeast extract derived sugar in presence of ManNAc, gene expression data showed a significant induction of the satABC SPG1589-91 and SPG1592 PTS transporters, and a non-significant induction of nanA (Figure 3C). We performed a second experiment that compared the influence of ManNAc at $\mathrm{OD}_{590}=0.02$ and 0.05 on gene expression (Figure 3B, open arrows). This assay compared bacteria grown on yeast-derived sugar and ManNAc, where the first time point represents growth on yeast-extract derived sugar (in presence of ManNAc) and the second growth on ManNAc after termination of the other carbohydrates. Growth on ManNAc caused a significant increase of transcriptional levels of all genes analysed (Figure 3D).
The values of mean fold changes were $17.61(\mathrm{p}<0.01)$ for nanA, $52.18(\mathrm{p}<0.01)$ for SPG1598, $6.33(\mathrm{p}<0.05)$ for SPG1592 and $6.65(\mathrm{p}<0.05)$ for satC SPG1591.

To evaluate the role of glucose and of the two amino sugars ManNAc and NeuNAc in the regulation of the $n a n A B$ regulon, we quantify gene expression during growth in the presence of these sugars. Bacteria were grown in the presence of ManNAc (Figure 4A, open triangles) or NeuNAc (Figure 4B, open triangles) and their gene expression was compared to that of bacteria grown with $1 \mathrm{~g} / \mathrm{L}$ glucose alone (Figure $4 \mathrm{~A}, \mathrm{~B}$, closed circles). All genes of the $\operatorname{nan} A B$ regulon showed a significant increase in transcription in presence of any of the amino sugars. The values of mean fold changes were: nanA, $2.69(\mathrm{p} \leq 0.05)$ in ManNAc and 5.14 $(\mathrm{p} \leq 0.05)$ in NeuNAc; SPG1598, $3.35(\mathrm{p} \leq 0.05)$ in ManNAc and 1.99 in NeuNAc; SPG1592, $3.21(\mathrm{p} \leq 0.05)$ in ManNAc and 3.74 $(\mathrm{p} \leq 0.05)$ in NeuNAc; SPG1591, $3.45(\mathrm{p} \leq 0.05)$ in ManNAc and $5.13(\mathrm{p} \leq 0.01)$ in NeuNAc. Interestingly the 
Table 1 List of gene annotation in the nanAB locus

\begin{tabular}{|c|c|c|c|c|c|c|c|}
\hline Annotation & Figure $1 A^{*}$ & $\begin{array}{c}\text { S. pneumoniae } \\
\text { TIGR4 }\end{array}$ & $\begin{array}{c}\text { S. pneumoniae } \\
\text { G54 }\end{array}$ & $\begin{array}{c}\text { S. mitis } \\
\text { B6 }\end{array}$ & $\begin{array}{l}\text { S. oralis } \\
\text { U05 }\end{array}$ & $\begin{array}{l}\text { S. gordonii } \\
\text { V288 }\end{array}$ & $\begin{array}{l}\text { S. sanguinis } \\
\text { SK36 }\end{array}$ \\
\hline Regulator & 1 & SP1674 & SPG1583 & smi0612 & SOR0560 & SG00127 & SSA0081 \\
\hline Hypothetical protein & 2 & - & - & smi0610 & SOR0559 & SGO0126 & SSA0080 \\
\hline $\mathrm{N}$-acetylmannosamine kinase & 3 & SP1675 & SPG1584 & smi0609 & SOR0558 & SGO0125 & SSA0079 \\
\hline $\mathrm{N}$-acetylneuraminate lyase & 4 & SP1676 & SPG1585 & smi0608 & SOR0557 & SGO0124 & SSA0078 \\
\hline hypothetical protein & 5 & SP1677 & SPG1586 & smi0607 & SOR0556 & - & - \\
\hline hypothetical protein & 6 & SP1679 & - & - & - & - & - \\
\hline hypothetical protein & 7 & SP1680 & SPG1588 & smi0606 & SOR0555 & - & - \\
\hline satA $A B C$ transporter permease & 8 & SP1681 & SPG1589 & smi0605 & SOR0553 & - & - \\
\hline satB $A B C$ transporter permease & 9 & SP1682 & SPG1590 & smi0604 & SOR0552 & - & - \\
\hline satC $A B C$ transporter substrate-binding protein & 10 & SP1683 & SPG1591 & smi0603 & SOR0550 & - & - \\
\hline PTS system, IIBC components & 11 & SP1684 & SPG1592 & - & - & - & - \\
\hline NanE, ManAc-6P 2-epimerase & 12 & SP1685 & SPG1593 & smi0602 & SOR0549 & SGO0118 & SSA0071 \\
\hline oxidoreductase & 13 & SP1686 & SPG1594 & - & - & SGO0123 & SSA0077 \\
\hline NanB neuraminidase & 14 & SP1687 & SPG1595 & - & - & - & - \\
\hline$A B C$ transporter permease & 15 & SP1688 & SPG1596 & - & - & SGO0122 & SSA0076 \\
\hline ABC transporter permease & 16 & SP1689 & SPG1597 & - & - & SGO0121 & SSA0075 \\
\hline$A B C$ transporter substrate-binding protein & 17 & SP1690 & SPG1598 & - & - & SGO0120 & SSA0074 \\
\hline hypothetical protein & 18 & SP1691 & SPG1599 & - & - & SGO0119 & SSA0073 \\
\hline NanA neuraminidase & 19 & SP1693 & SPG1600 & smi0601 & SOR0548 & - & - \\
\hline Acetyl xylan esterase & 20 & SP1694 & SPG1601 & smi0600 & SOR0547 & - & SSA0070 \\
\hline
\end{tabular}

${ }^{*}$ numbers as in Figure $1 \mathrm{~A}$.

transporter SPG1596-8 linked to the growth and fermentation of ManNAc was more induced by this sugar, while NeuNAc had a significantly greater effect on the satABC SPG1589-91 transporter, again in accordance with phenotypic data.

Repression of the nan $A B$ locus in the presence of glucose According to the presence of three cre sites within the pneumococcal neuraminidase locus, we observed a biphasic growth curve when bacteria grew on glucose plus ManNAc or NeuNAc (Figure 4A,B, open squares). To demonstrate that this phenotype was due to carbon catabolite repression, we investigated the transcriptional behaviour of the neuraminidase locus in the presence or absence of glucose in the medium. Growth conditions used were as follows: ManNAc with and without glucose (Figure 4A, open triangles and open squares), NeuNAc with and without glucose (Figure 4B, open triangles and open diamonds) and glucose as the sole carbon source as a reference condition (Figure $4 \mathrm{~A}$ and $4 \mathrm{~B}$, closed circles). Growth curve data show that addition of glucose to both ManNAc and NeuNAc resulted in an initial growth on glucose as a preferred carbon source followed by a second slower growth phase, in which the amino sugars were metabolised. To assess glucose repression during growth on glucose gene expression analysis was carried out by sampling the bacteria at an $\mathrm{OD}_{590}$ of 0.05 (Figure $4 \mathrm{~A}, \mathrm{~B}$, arrows). As shown in Figure $4 \mathrm{C}$, the overexpression of all genes of the $\operatorname{nan} A B$ locus occurred during growth on ManNAc or NeuNAc as the sole carbon sources (Figure 4C, open and grey bars), while it was completely repressed in the presence of glucose (Figure 4C, striped bars).

\section{Regulation of neuraminidase A production and activity by ManNAc}

To assess the production of NanA on the bacterial surface after induction of the $\operatorname{nan} A B$ locus by ManNAc or NeuNAc, we performed a cytofluorimetry assay. In these experiments bacteria were harvested at the late exponential phase. In this assay the anti-NanA serum recognises also to a certain extent glucose grown bacteria (Figure 5A). However in culture media with either ManNAc or NeuNAc as the sole carbon sources, the number of NanA expressing bacterial cells significantly increased reaching $73.7 \%( \pm 3.4)$ and $79.6 \%( \pm 4.9)$, respectively. Differences in NanA production between bacterial cells grown with either of the two amino sugars and control cells cultured in glucose or glucose plus ManNAc were statistically significant (Figure 5A). For more detailed analysis a fluorescence assay for the detection of cell surface-associated neuraminidase activity was carried 

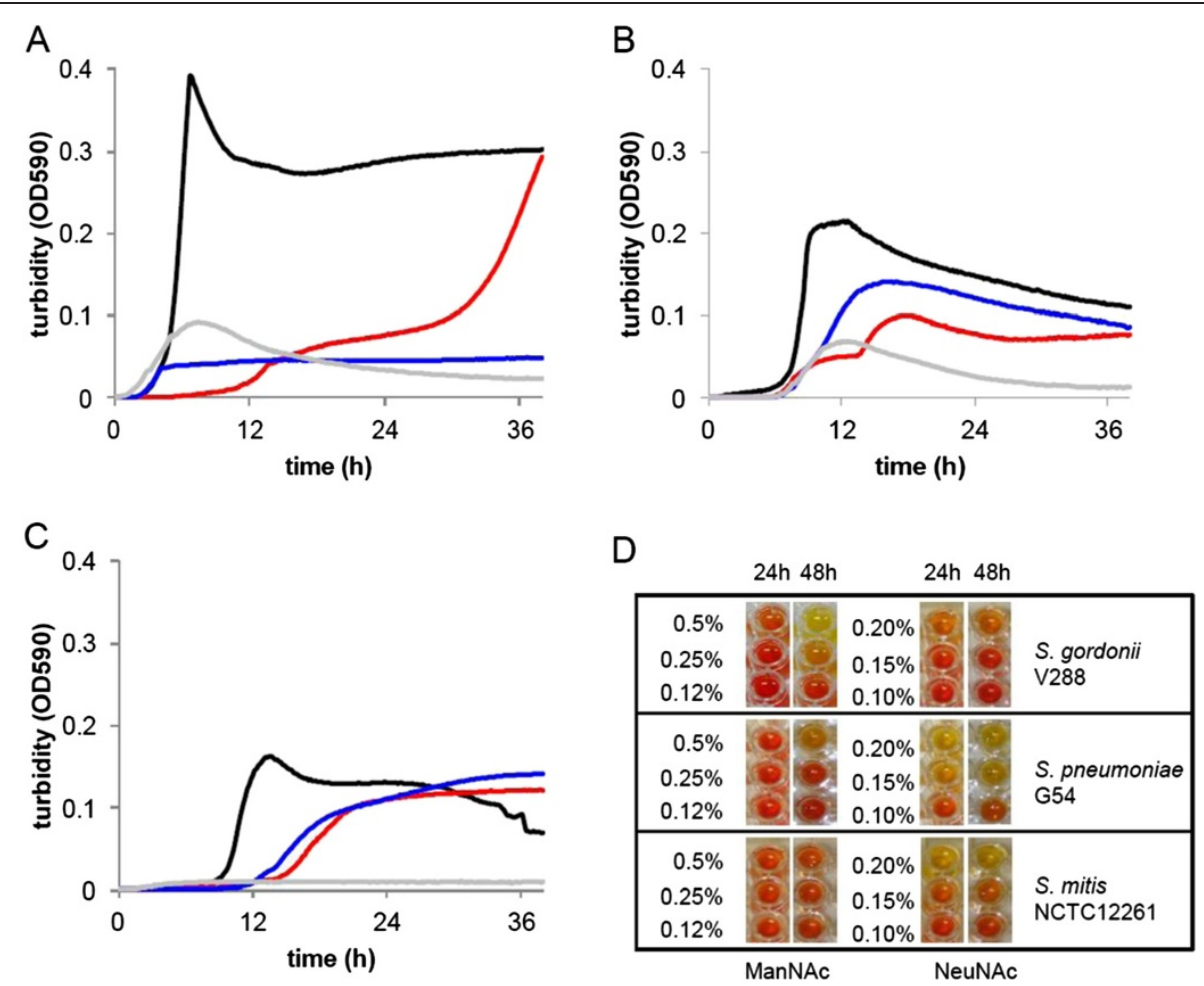

Figure 2 Metabolic utilisation of ManNAc and NeuNAc by S. gordonii, S. mitis and S. pneumoniae. S. gordonii V288 (A), S. pneumoniae G54 (B), and S. mitis NCTC12661 (C) were grown in CAT medium (200 U catalase) supplemented with $2 \mathrm{~g} / \mathrm{L}$ glucose (black line) $2 \mathrm{~g} / \mathrm{L}$ ManNAc (red line) and $1.5 \mathrm{~g} / \mathrm{L}$ NeuNAc (blue line). CAT medium alone as a source of carbon is in grey line. All strains were grown for 38 hours at $37^{\circ} \mathrm{C}$ in $200 \mu \mathrm{l}$ of medium in a 96 well microplate with reading intervals of $10 \mathrm{~min}$. For the fermentation assay (panel D) bacteria were incubated for 24 and $48 \mathrm{~h}$ with serial dilutions of either ManNAc (left columns) or NeuNAc (right columns) as sole carbon sources in microtiter plates containing phenol red as a pH indicator. Sugar fermentation is evidenced by a yellow colour change due to acidification of the culture medium. Carbohydrate concentrations (\% w/v) are shown on the right.

out to investigate the dependence of neuraminidase production upon the nature of the carbon source provided during bacterial growth (glucose vs N-acetylmannosamine). The amount of neuraminidase activity in cell samples containing $10^{7} \mathrm{CFU} / \mathrm{ml}$ was clearly higher (approx. 12-fold) in the presence of $\mathrm{N}$-acetylmannosamine rather than glucose (Figure 5B), indicating that $\mathrm{N}$ acetylmannosamine is an inducer of neuraminidase production while in glucose grown cells neuraminidase activity is clearly repressed. $10^{7} \mathrm{CFU}$ of $\mathrm{S}$. pneumoniae FP65 grown in the presence of $\mathrm{N}$-acetylmannosamine yielded a neuraminidase activity equivalent to that of $7.5 \mu \mathrm{g}$ of purified NanA, indicating that this strain produces a significant amount of neuraminidase(s) in the presence of amino sugars. These numbers are such to propose approximately 100-500 enzymes per cell when bacteria are grown in amino sugar and only few enzymes per cell when bacteria are grown in glucose.

\section{Discussion}

Pneumococcal neuraminidases are the most studied surface located glycosyl-hydrolases due to their role in pathogenicity as factors involved in adhesion and invasion of
S. pneumoniae to host cells $[5,6,8,9,27,28]$. In addition, their role in the release of free sialic acid from oligosaccharides has been proposed as an important source of carbon and energy [13,14,29,30]. More recently, the cleavage of sialic acid from O-glycans has been related to pathogenesis, by proposing sialic acid as a molecular signal to promote in vitro biofilm production and in vivo nasopharyngeal carriage and lung invasion by S. pneumoniae $[10,17]$. In this context, the regulatory mechanisms of the neuraminidase locus expression are of importance. So far nearly all data on virulence and expression of the two loci containing neuraminidases has been carried out on the $n a n A B$ locus only, since the D39 reference strain does not carry the $n a n C$ locus [18]. The main finding on expression of the $n a n A B$ locus reported its organisation in four predicted transcriptional units, of these the one harbouring NanA and the one encoding for the enzymes of the sialic acid metabolism were differentially expressed in transparent and opaque pneumococcal colony variants [21]. Additionally the increased expression of this locus during infection $[10,24,25]$, further underlines the importance of neuraminidases in the interaction of pneumococci with the host. It should be noted that most 

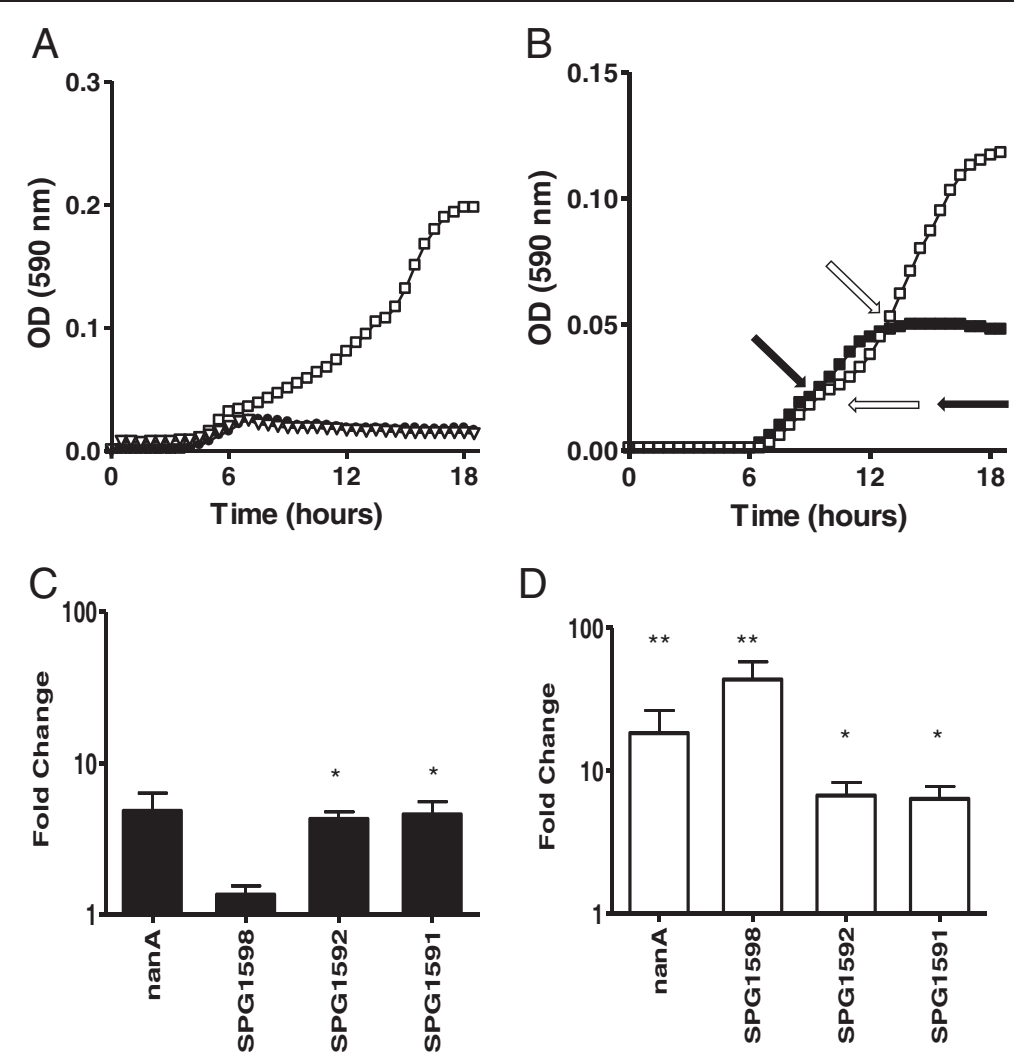

Figure 3 Growth and induction of gene expression by ManNAc. (A) Growth of S. pneumoniae strains on CAT medium supplemented with $10 \mathrm{~g} / \mathrm{L}$ of ManNAc: FP65 (open squares), nanAB-deficient mutant (open triangles), and SPG1583-regulator deletion mutant (closed circles). (B) Growth of FP65 on CAT medium without added sugar (closed squares) and supplemented with ManNAc $10 \mathrm{~g} / \mathrm{L}$ (open squares). The white and black arrows indicate samples taken for quantitative Real Time-PCR. Gene expression analysis of the genes coding for NanA the ABC transporter SPG1598, the PTS transporter SPG1592, and the ABC transporter SPG1591 is shown in panel $\mathbf{C}$ and $\mathbf{D}$. Panel $\mathbf{C}$ refers to fold changes in transcriptional levels at OD 0.02 in medium with or without ManNAc (for sampling see closed arrows in panel 3B). Panel D refers to analysis of sequential samples $\left(\mathrm{OD}_{590}=0.02\right.$ and $\left.\mathrm{OD}_{590}=0.05\right)$ of bacteria grown in ManNAc (for sampling see open arrows in panel $3 \mathbf{B}$ ). The fold changes are reported as mean from independent triplicate or quadruplicate experiments. Two-tailed Student t test was used for analyse statistical significance $\left(*, p<0.05 ;{ }^{* *}, p<0.01\right)$. Generation time on unsuplemented CAT medium is 40 min and on ManNAc 140 min.

of the above work on pneumococcal virulence is done utilising strain D39, which is unable to ferment sialic acid due to a frame shift in the neuraminate lyase of the nan $A B$ locus [23,31], a fact which apparently does not influence regulation of the locus and virulence of the bacterium.

We have recently shown that the two $A B C$ transporters of the $\operatorname{nan} A B$ locus, and also the sodium symporter of the nanC locus to a lesser extent, are not only involved in sialic acid uptake, but also in the transport of ManNAc, which represents the first metabolic intermediate in pneumococcal NeuNAc catabolism [23]. In this work we focus our attention on the contribution of the $\operatorname{nan} A B$ locus, since deletion mutants for the nanC locus had been shown not to influence growth on ManNAc and NeuNAc during the first 18-24 hours of incubation, implying a limited or absent regulatory crosstalk between the two regulons $[14,23]$. The two ABC transporters were shown to be able to support growth on amino sugars, with SPG1596-8 and SPG1589-91 being the main transporters for ManNAc and NeuNAc, respectively [23]. In this work we have combined genomic information, gene expression and growth phenotypes to further clarify these data. When performing in silico analysis of the $n a n A B$ locus we observed the presence of part of the locus in related oral streptococci. Here we utilised this genomic information to strengthen the correlation between orthologous transporters and metabolic functions. S. sanguinis and S. gordonii, harbouring an operon including the orthologue of the SPG1596-8, were found to be able to efficiently metabolise ManNAc, but not NeuNAc. To the contrary S. mitis and S. oralis, which are much more closely related to pneumococci, harboured a locus, in addition to all the metabolic genes, also encoding for a neuraminidase and the orthologue of the satABC SPG1589-91 transporter [14]. The finding that $S$. mitis can efficiently metabolise NeuNAc and ManNAc, confirm that the substrate specificity identified 


\section{A}
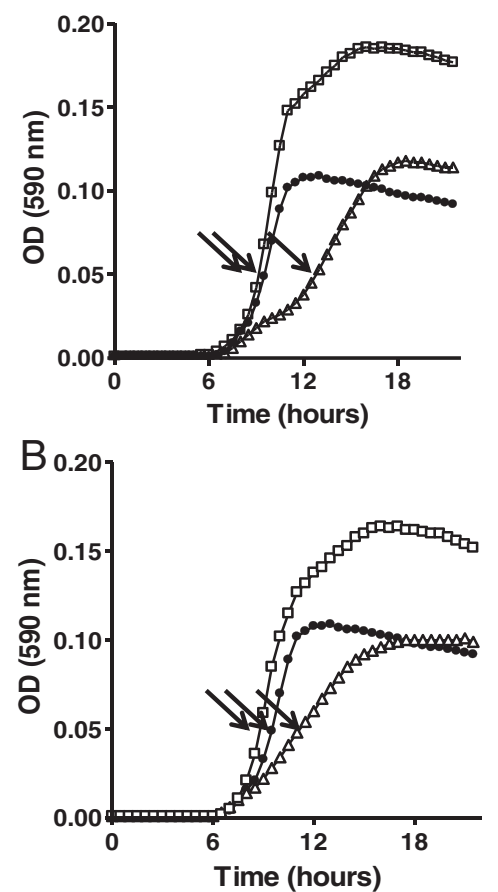

C

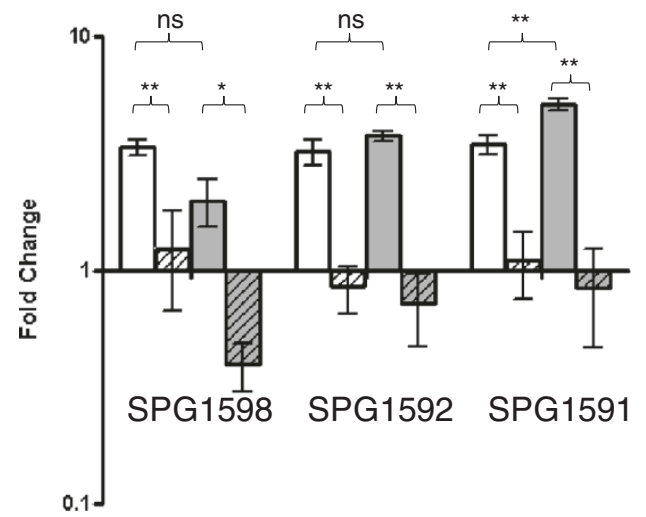

Figure 4 Repression of nanAB locus by glucose. (A) Growth curves of FP65 in medium supplemented with glucose (closed circles), ManNAC (open triangles), and glucose plus ManNAc (open squares). (B) Growth curves of PF65 in medium supplemented with glucose (open circles), NeuNAc (open triangles), and glucose plus NeuNAc (open squares). The arrows indicated sampling. (C) Gene expression of SPG1598, SPG1592, and SPG1591 in medium supplemented with amino sugars are compared to growth in glucose. Variation of gene expression is shown for genes of bacteria grown in ManNAc (open bars), glucose plus ManNAc (open striped bars), NeuNAc (grey bars), and glucose plus NeuNAc (grey striped bars). Results are represented as fold changes \pm SD of gene expression from 3 to 4 independent experiments. Statistical analysis was carried out using Tukey's Multiple Comparison Test (ns non significant; ${ }^{*}, \mathrm{p}<0.05 ;{ }^{* *}, \mathrm{p}<0.01$ ). Generation time on glucose containing media is $38-45$ min, 90 min on NeuNAc and 140 min on ManNAc.

for the pneumococcal transporters is generally well conserved in orthologues of related species [14]. Interestingly, all oral streptococci share the core part of metabolic enzymes of the operon, suggesting comparable capability to metabolise both NeuNAc and ManNAc. These observations match earlier data that described detectable levels of metabolism of NeuNAc in most oral streptococci, while sialidase activity could only be found in few species [32]. Amongst the oral streptococci, pneumococci carry a composite locus, probably assembled from the gene pool of related species. The association of the SPG1594 oxidoreductase with ManNAc metabolism and of two small hypothetical proteins (SPG1586 and SPG1588) with NeuNAc metabolism remains unexplained, as all necessary enzymes for sialic acid metabolism appear to be already present. The PTS transporter, found to transport glucosamine, appears to be unique in pneumococci [23]. The fact that glucosamine is the last metabolic intermediate in sialic acid catabolism may indicate a convenience for the bacterium in co-utilisation of $\mathrm{GlcN}$ and ManNAc, even if it is not clear where pneumococci should feed on GlcN, a rare sugar in the human nasopharynx, but of which on the contrary the pneumococcal cell wall is exceptionally rich [33].

When pneumococci grow on ManNAc and NeuNAc as the sole carbon sources, the generation time is much longer than on glucose or on the yeast-extract derived carbohydrates of the CAT medium, which is in accordance with previous data [23]. Growth on ManNAc (Figure 3B, Figure 4A) shows a profile with a change in generation time. In the case of growth on glucose repression of the whole locus indicates sequential utilisation of sugars. This is less clear for the growth on yeast extract derived dextran and ManNAc, where only part of the locus is induced with the exception of the predicted central transcriptional unit encoding the principal ManNAc ABC transporter SPG1596-8. The data here presented thus do not rule out, that during growth on yeast derived sugars also ManNAc may be co-metabolised. The differential impact of regulation on the three operons is reminiscent of data on expression of this locus in transparent colony variants, where also the $n a n B$ and ManNAc-uptake operon is not involved in differential expression, while the other two transcripts 


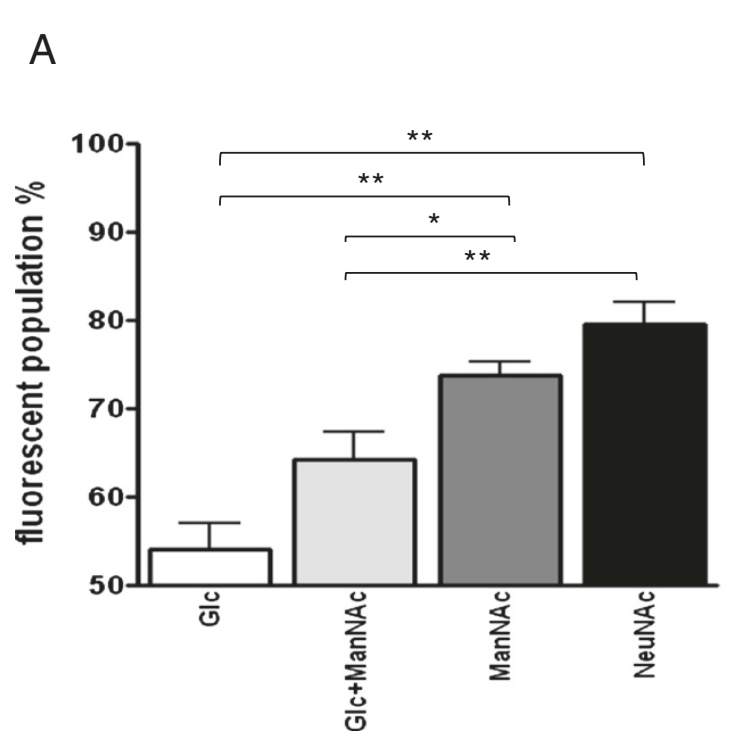

\section{B}

Figure 5 Neuraminidase protein production and activity on whole cells. A cytofluorimetric assay with an anti-NanA serum was performed on pneumococci grown on different carbohydrates (panel $\mathbf{A}$ ). The presence of NanA at the bacterial surface was tested in samples cultivated in glucose (open bar), glucose + ManNAc, ManNAc alone (grey bar), and NeuNAc alone (black bar) (all carbohydrates were at $1 \mathrm{~g} / \mathrm{L}$ ). Data are represented as mean values \pm SD of percent bacterial population positive for NanA production and derived from quadruplicates experiments performed independently. Asterisks ( $\left.{ }^{*}, p<0.05 ;{ }^{* *}, p<0.001\right)$ indicated statistical significance. Panel $\mathbf{B}$ shows the hydrolysis of 2'-(4-Methylumbelliferyl)-a-D-N-acetylneuraminic acid (4MU-Neu5Ac) in the presence of $40 \mu \mathrm{l}$ S. pneumoniae FP65 cell samples grown in CAT medium with either glucose (white circles) or $\mathrm{N}$-acetylmannosamine (black circles). The neuraminidase activity was computed as the variation of fluorescence vs time using a linear regression of the data (dashed lines). Inlet. Hydrolysis of 4MU-Neu5Ac by purified NanA neuraminidase, showing the proportionality between enzyme concentration and rate of fluorescence variation. Enzyme concentrations were $10 \mathrm{nM}$ (black circles), $20 \mathrm{nM}$ (triangles), $30 \mathrm{nM}$ (diamonds) and $40 \mathrm{nM}$ (squares). The empty circles show the variation of fluorescence vs time for the substrate alone.

are upregulated [21]. The fact that both ManNAc and NeuNAc are able to efficiently induce the operon is in accordance with our finding that the SPG1583 regulator acts a positive regulator, as documented by absence of metabolism in its mutant and also by its annotation as a phosphor-sugar binding regulator. Since NeuNAc is imported by an $\mathrm{ABC}$ transporter, which does not phosphorylate during uptake, and is first hydrolysed to ManNAc before becoming phosphorylated (Figure 1B), both amino sugars may equally originate the inducer of the positive regulator; probably ManNAc-phosphate.

The sequential utilization of carbon sources is generally regulated by carbon catabolite repression, and in bacteria it has been linked not only to metabolic use, but also to more general mechanisms involved in hostpathogen interactions [34,35]. As in other Gram-positive bacteria, also in S. pneumoniae carbon catabolite repression involves the catabolite control protein A (CcpA) which regulates operons by binding to a specific operator sequence, named as catabolite-repressible element (cre site) [36-39]. Multiple cre sites were recently predicted upstream SPG1601, SPG1597 and SPG1593 in the $\operatorname{nan} A B$ locus $[37,38]$, and array analyses proved the role of CcpA in its regulation and interestingly relief of ссрA repression shows much more pronounced effects on the "NeuNAc-operon" (SPG1593-84) than on the
"ManNAc operon" (SPG1599-4). The cre sites and CcpA-mediated regulation is in accordance with the transcriptional units described earlier [21]. Our data here confirm that glucose completely represses the expression of all three predicted transcriptional units of the $n a n A B$ locus. The above gene expression data are also consistent with the neuraminidase activity assay on whole cells, which indicates twelve times more enzymatic activity in induced cells with respect to glucose grown cells. The repression of both neuraminidases and the intracellular enzymes for sialic acid metabolism had already been reported for a large number of viridians streptococci, which thus share with S. pneumoniae a strong effect of carbon catabolite repression on the loci responsible of NeuNAc metabolism [32].

\section{Conclusions}

In summary, the data obtained in our study confirmed and demonstrated that, (i) pneumococci carry a composite locus, in part shared by related species, which is predicted to metabolise both ManNAc and NeuNAc, (ii) pneumococci could use both ManNAc and NeuNAc as the sole carbon sources for growth, (iii) uptake of ManNAc and NeuNAc involved preferentially the SPG1596-8 and the satABC SPG1589-91ABC transporters, respectively, (iv) ManNAc and NeuNAc could 
induce the $n a n A B$ locus, which is subjected to carbon catabolite repression by glucose and (v) a quantitative neuraminidase activity assay allowed to tentatively quantify neuraminidases on the surface of pneumococci grown in amino sugars to numbers around 100-500 enzymes per cell. Interestingly, some growth conditions were found to mimic the transcriptional profile observed for pneumococcal transparent colony variants, suggesting a metabolic influences on pneumococcal phase variation [21]. Still, the differential induction of the predicted transcriptional units by the two amino sugars, indicates that probably carbon catabolite repression and activation by the regulator act at different strength on the three transcriptional units. Finally as already shown in oral streptococci [32], the amount of NanA significantly increases and neuraminidase activity during growth on ManNAc or NeuNAc, indicating that experimental conditions based on mid log glucose-grown bacterial cells may be biased in estimating the actual contribution of neuraminidases to host-pathogen interaction.

\section{Methods}

\section{Bacterial strains and culture media}

Bacterial strains used in this work were S. pneumoniae strain G54 (serotype 19F) and its un-encapsulated derivative FP65 [40], since the pneumococcal reference strain D39 has a frame shifted neuraminate lyase gene and TIGR4 did not grow efficiently in CAT medium [23]. Most experiments are performed with the un-encapsulated FP69 as strains without are non virulent and no influence on sugar metabolism has been observed (data not shown). Oral streptococci where $S$. mitis NCTC12261 (kindly provided by Morgens Kilian) and S. gordonii V288 Challis [41]. Bacteria were plated on Tryptic soy agar plates (TSB; Liofilchem Roseto degli Abruzzi, Italy) containing 3\% v/v of horse blood. Stocks grown in TSB at $37^{\circ} \mathrm{C}$ to $\mathrm{OD}_{590}$ of 0.2 were supplemented with $20 \%$ glycerol and stored at $-80^{\circ} \mathrm{C}$. For fermentation assays and growth curves, bacteria were grown in CAT medium composed of bacto casitone $10 \mathrm{~g} / \mathrm{l}$ (Becton Dickinson), bacto yeast extract $1 \mathrm{~g} / \mathrm{l}$ (Becton Dickinson), tryptone $5 \mathrm{~g} / \mathrm{l}$ (Oxoid Hampshire, UK) and sodium chloride $5 \mathrm{~g} / \mathrm{l}$ [42]. Just before use, CAT medium was supplemented with $3 \% \mathrm{w} / \mathrm{v}$ of $\mathrm{K}_{2} \mathrm{HPO}_{4} 0.5 \mathrm{M}$ [43], a carbon source and catalase $200 \mathrm{U} / \mathrm{ml}$. The sugars were glucose (Sigma-Aldrich), N-Acetylneuraminic acid (NeuNAc, Carbosynth, Compton, UK) and N-Acetyl-D-mannosamine (ManNAc, Carbosynth, Compton, UK). Due to the presence of bacto-yeast extract (Beckton Dickinson), the carbohydrate non-supplemented CAT medium contained $0.16 \mathrm{~g} / \mathrm{l}$ of total carbohydrate.

\section{Mutant construction}

Mutants were constructed by direct transformation of S. pneumoniae with PCR generated recombinant DNA fragments [43]. For deletion of the whole nanAB locus, primers NanA1 (TGTAGCCGTCATTTTATTGCTAC), NanA2 (TCCACTAGTTCTAGAGCGATTTTCTGCCT GATGTTGGTAT), NanA3 (ATCGCTCTTGAAGGGA ATGCTATTTACACCATACTTCCT), and NanA4 (CA GCTTCGCCTTGCCGTAGGT) were used to amplify segments to allow the integration of the spectinomycin marker aad 9 and the deletion of the whole $n a n A B$ locus (SPG1583 -SPG1600). For deletion of the SPG1583 regulator, primers DC_09 (TGTCTACGATAGCCGTTGAG), DC_10 (ATCAAACGGATCCCCAGCTTGAACCAGC ATCATGGATGAAAATTG), DC_11 (ATATTTTACTG GATGAATTGTTTTAGAAAGCCGTCTTGGTCTGTC), and DC_12 (AATCGCTCGCTATTTTTTGC) were used to amplify segments to allow the substitution of the kanamycin maker aphIII with the whole nanAB locus, as previously described [44].

\section{Bioinformatic tools}

Comparative genomic analysis was performed using the ACT (Artemis Comparison Tool) [45]. Genbank files for sequence comparison were downloaded directly from the NCBI website. The $S$. pneumoniae genomes utilised were of strain TIGR4 and G54 (NC_003028 and NC_011072). The genomes used for comparison were from $S$. gordonii strain Challis (NC_009785) [46], S. mitis strain B6 [47] (NC_013853), S. oralis strain Uo5 (NC_015291) [48], and S. sanguinis strain SK36 (NC_009009) [49].

\section{Carbohydrate fermentation}

The method for evaluation sugar uptake and fermentation has been recently described [23]. Briefly, bacteria grown on agar plates were resuspended to an $\mathrm{OD}_{590}=$ 0.6 in CAT medium and diluted 1:1 with CAT medium supplemented with $\mathrm{K}_{2} \mathrm{HPO}_{4}$, the appropriate sugar and catalase as reported above. After o.n. incubation, $\mathrm{pH}$ changes were visualised by addition of phenol red $(0,1 \mathrm{mg} / \mathrm{ml})$ (P4633 Sigma-Aldrich).

\section{Growth curve and sample collection}

In order to characterize the gene expression pattern in a specific point of the growth curve, we sampled bacteria during growth. Strains were grown on TSA plates at $37^{\circ} \mathrm{C}$ in a $\mathrm{CO}_{2}$ enriched atmosphere for 18 hours. Bacteria were then collected with a swab and resuspended at the $\mathrm{OD}_{590}$ of 0.2 in non-supplemented CAT medium. Bacterial samples were diluted 1:100 in CAT medium either without added sugar or with addition of either glucose, ManNAc, NeuNAc, glucose + ManNAc, or glucose + NeuNAc, all at $1 \mathrm{~g} / \mathrm{L}$. Bacterial growth curves were performed in 96-well plates in a thermostated spectrophotometer at $37^{\circ} \mathrm{C}$. Plates were shaken gently for 10 seconds prior to each reading, and the optical density was read automatically in $10 \mathrm{~min}$ intervals 
at a wave length of $590 \mathrm{~nm}$. Triplicate samples were collected from microwells for gene expression analysis and cytofluorimetry. For RNA extraction and retrotranscription, the samples were transferred to microtubus, centrifuged at $13000 \mathrm{rpm}$ at $4^{\circ} \mathrm{C}$ for $1 \mathrm{~min}$, and the pellet was conserved at $-20^{\circ} \mathrm{C}$. For flow-cytometry analysis, the samples were centrifuged at $8000 \mathrm{rpm}$ at room temperature for $5 \mathrm{~min}$ and immediately analysed.

\section{RNA extraction, retrotranscription and qPCR}

RNA was extracted using the NucleoSpin RNA II kit (Macherey-Nagel) according to the manufacturer's instructions, and the RNA samples were frozen in aliquots until use. cDNA synthesis was carried out using the Transcriptor First strand cDNA synthesis kit (Roche) according to the manufacturer's instructions. Annealing was performed at $25^{\circ} \mathrm{C}$ for $10 \mathrm{~min}$, extension at $37^{\circ} \mathrm{C}$ for $1 \mathrm{~h}$, and finally inactivation at $70^{\circ} \mathrm{C}$ for $15 \mathrm{~min}$. The qPCR was performed as previously described [50], by mixing $2 \mu \mathrm{l}$ of cDNA template, $10 \mathrm{pmol}$ of primers, and $2 \mu \mathrm{l}$ of Light Cycler DNA-Master SYBR Green I (Roche). The reaction was carried out in a Light Cycler apparatus (Roche). Primer efficiency was verified by serial dilution of cDNA ranging from $10^{2}$ to $10^{6}$ target copies per reaction. Primers were designed on $\operatorname{gyr} B$ (reference gene; CAGATCAAGAAATCAAACTCCAA and CAGCAT CATCTACAGAAACTC), nanA SPG1600 (AGCAACCT CTGGCAAATGAA and ATAGTAATCTCTTGGAATT), SPG1598 (GGTCAACTCAGATGCTT and GAGGAA CAGAGTAGTAATC), SPG1592 (CCAACCACGATAG CAAC and CTGAATACAACCTCTCC) and SPG1591 (CAGGTGCTTTCCCAGTC and GTGTTGTAGTATGG TGAG) [24,50]. The relative gene expression was analysed by using the $2^{-\Delta \Delta C T}$ method [51]. At least three replicas were used for any given sample. Statistical analysis was conducted by using the two-tailed Student $t$ test.

\section{Flow cytometry assay}

FP65 pneumococci grown in media with carbohydrate supplementations at $1 \mathrm{~g} / \mathrm{L}$ to late $\log$ phase were resuspended in $500 \mu \mathrm{l}$ of phosphate-buffered saline (PBS; pH 7.4) containing $1 \%$ bovine serum albumin and incubated at $37^{\circ} \mathrm{C}$ for $30 \mathrm{~min}$. Samples were spun down and pellets were resuspended in anti-NanA rabbit serum diluted 1:100 in $\mathrm{PBS} / \mathrm{BSA}$ and incubated at $4^{\circ} \mathrm{C}$ for $1 \mathrm{~h}$ (negative controls were incubated without antibody). After two washes with $1 \mathrm{ml}$ of PBS, $100 \mu \mathrm{l}$ of fluorescein isothiocyanate (FITC)conjugated anti-rabbit (1:64; Sigma-Aldrich) was added to bacterial pellets. The resuspensions were incubated at $37^{\circ} \mathrm{C}$ for $30 \mathrm{~min}$ and then washed twice in PBS. Samples were finally resuspended in $300 \mu \mathrm{l}$ of paraformaldehyde $1 \%$ in PBS and subjected to flow cytometry (FACScan, Becton Dickinson, San Diego, CA). Statistical analysis was carried out by using two-tailed Student $t$ test.

\section{Neuraminidase activity}

The neuraminidase activity was measured using the fluorogenic substrate 2 -(4-methylumbelliferyl)- $\alpha$-D-Nacetylneuraminic acid (4MU-Neu5Ac) (M8639, SigmaAldrich, St. Louis, Miss.). The time dependence of the variation of fluorescence ( $\lambda_{\text {excitation }}, 335 \mathrm{~nm} ; \lambda_{\text {emission }}$, $400 \mathrm{~nm}$ ) in the presence of cell or enzyme samples was recorded with a EnVision multilabel plate reader (Perkin Elmer, Waltham, Mass.) using $50 \mu \mathrm{M}$ 4MUNeu5Ac in 10 mM MES buffer at $\mathrm{pH} 6.0$, in a final reaction volume of $200 \mu \mathrm{l}$. S. pneumoniae FP65 was grown in CAT medium, containing alternatively glucose or Nacetylmannosamine as the carbon source, respectively, for 18 hours at $37^{\circ} \mathrm{C}$. The sample was prepared as follows; the culture was centrifuged at $10,000 \times \mathrm{g}\left(4^{\circ} \mathrm{C}\right)$ and the cell pellet washed once in an equal volume of 10 MES buffer $\mathrm{pH}$ 6.0, centrifuged and resuspended at a final $\mathrm{A}^{600}=0.4$ in $10 \mathrm{mM}$ MES $\mathrm{pH}$ 6.0. The method was initially optimized and calibrated using purified NanA neuraminidase of S. pneumoniae D39 produced in E. coli $(0.88 \mathrm{mg} / \mathrm{ml})$ (data not shown). The activity was computed as the variation of fluorescence $v s$ time using a linear regression of the data. In our conditions, $1 \mu \mathrm{g}$ of purified NanA yielded a activity of 10,690 $\Delta \mathrm{F} / \mathrm{min}$.

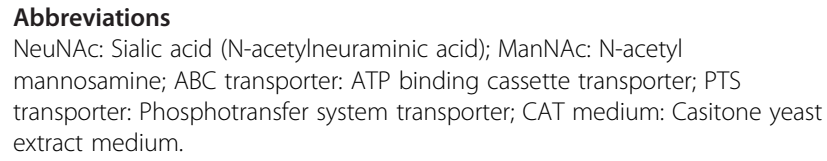

\section{Acknowledgements}

The work was in part funded by the European Commission grant PNEUMOPATH FP7-HEALTH-222983 and by Ricerca Regionale Toscana in Materia di Salute 2009-201.

\section{Author details}

${ }^{1}$ Dipartimento di Biotecnologie, Università di Siena, Siena, Italy. ${ }^{2}$ Department of Infection Immunity and Inflammation, University of Leicester, Leicester, United Kingdom. ${ }^{3}$ UOC Batteriologia, Azienda Universitaria Ospedaliera Senese, Siena, Italy. ${ }^{4}$ LAMMB, Policlinico Le Scotte lotto 5 piano 1, Siena 53100, Italy. ${ }^{5}$ Research Centre for Infectious Diseases, School of Molecular and Biomedical Science, University of Adelaide, South Australia 5005, Australia.

Received: 13 February 2012 Accepted: 5 September 2012 Published: 11 September 2012

\section{References}

1. Kadioglu A, Weiser JN, Paton JC, Andrew PW: The role of Streptococcus pneumoniae virulence factors in host respiratory colonization and disease. Nat Rev Microbiol 2008, 6:288-301.

2. King SJ: Pneumococcal modification of host sugars: a major contributor to colonization of the human airway? Mol Oral Microbiol 2010, 25:15-24. 
3. Camara M, Boulnois GJ, Andrew PW, Mitchell TJ: A neuraminidase from Streptococcus pneumoniae has the feature of a surface protein. Infect Immun 1994, 62:3688-3695.

4. Berry AM, Paton JC: Sequence heterogenicity of PsaA, a 37-kilodalton putative adhesin essential for virulence of Streptococcus pneumoniae. Infect Immun 1996, 64:5255-5262.

5. McCullers JA, Bartmess KC: Role of neuraminidase in lethal synergism between influenza virus and Streptococcus pneumoniae. J Infect Dis 2003, 187:1000-1009.

6. Manco S, Hernon F, Yesilkaya H, Paton JC, Andrew PW, Kadioglu A Pneumococcal neuraminidases $A$ and $B$ both have essential roles during infection of the respiratory tract and sepsis. Infect Immun 2006 74:4014-4020.

7. Tong HH, James M, Grants I, Liu X, Shi G, DeMaria TF: Comparison of structural changes of cell surface carbohydrates in the eustachian tube epithelium of chinchillas infected with a Streptococcus pneumoniae neuraminidase-deficient mutant or its isogenic parent strain. Microb Pathog 2001, 31:309-317

8. Banerjee A, Van Sorge NM, Sheen TR, Uchiyama S, Mitchell TJ, Doran KS: Activation of brain endothelium by pneumococcal neuraminidase NanA promotes bacterial internalization. Cell Microbiol 2010, 12:1576-1588

9. Uchiyama S, Carlin AF, Khosravi A, Weiman S, Banerjee A, Quach D, et al: The surface-anchored NanA protein promotes pneumococcal brain endothelial cell invasion. J Exp Med 2009, 206:1845-1852.

10. Parker D, Soong G, Planet P, Brower J, Ratner AJ, Prince A: The NanA neuraminidase of Streptococcus pneumoniae is involved in biofilm formation. Infect Immun 2009, 77:3722-3730.

11. Johnston JW, Zaleski A, Allen S, Mootz JM, Armbruster D, Gibson BW, et al: Regulation of sialic acid transport and catabolism in Haemophilus influenzae. Mol Microbiol 2007, 66:26-39.

12. Rohmer $L$, Hocquet $D$, Miller SI: Are pathogenic bacteria just looking for food? Metabolism and microbial pathogenesis. Trends Microbiol 2011, 19:341-348.

13. Yesilkaya H, Manco S, Kadioglu A, Terra VS, Andrew PW: The ability to utilize mucin affects the regulation of virulence gene expression in Streptococcus pneumoniae. FEMS Microbiol Lett 2008, 278:231-235.

14. Marion C, Burnaugh AM, Woodiga SA, King SJ: Sialic acid transport contributes to pneumococcal colonization. Infect Immun 2011, 79:1262-1269.

15. Almagro-Moreno S, Boyd EF: Insights into the evolution of sialic acid catabolism among bacteria. BMC Genomics 2009, 26:118.

16. Vimr ER, Kalivoda KA, Deszo EL, Steenbergen SM: Diversity of microbial sialic acid metabolism. Microbiol Mol Biol Rev 2004, 68:132-153.

17. Trappetti C, Kadioglu A, Carter M, Athwal J, lannelli F, Pozzi G, et al: Sialic acid: a preventable signal for pneumococcal biofilm, colonisation and invasion of the host. J Infect Dis 2009, 199:1497-1505.

18. Pettigrew MM, Fennie KP, York MP, Daniels J, Ghaffar F: Variation in the presence of neuraminidase genes among Streptococcus pneumoniae isolates with identical sequence types. Infect Immun 2006, 74:3360-3365.

19. Xu H, Sullivan TJ, Sekiguci J, Kirikae T, Ojima I, Stratton CF, et al: Mechanism and inhibition of saFabl, the enoyl reductase from Staphylococcus aureus. Biochemistry 2008, 47:4228-4236.

20. Xu G, Kiefel MJ, Wilson JC, Andrew PW, Oggioni MR, Taylor GL: Three Streptococcus pneumoniae Sialidases: three different products. J Am Chem Soc 2011, 133:1718-1721.

21. King SJ, Hippe KR, Gould JM, Bae D, Peterson S, Cline RT, et al: Phase variable desialylation of host proteins that bind to Streptococcus pneumoniae in vivo and protect the airway. Mol Microbiol 2004, 54:159-171.

22. Almagro-Moreno S, Boyd EF: Bacterial catabolism of nonulosonic (sialic) acid and fitness in the gut. Gut Microbes 2010, 1:45-50.

23. Bidossi A, Mulas L, Decorosi F, Colomba L, Ricci S, Pozzi G, et al: A functional genomics approach to establish the complement of carbohydrate transporters in Streptococcus pneumoniae. PLoS One 2012, 7:e33320.

24. Oggioni MR, Trappetti C, Kadioglu A, Cassone M, lannelli F, Ricci S, et al: Switch from planktonic to sessile life: a major event in pneumococcal pathogenesis. Mol Microbiol 2006, 61:1196-1210

25. LeMessuier KS, Ogunniyi DA, Paton JC: Differential expression of key pneumococcal virulence genes in vivo. Microbiology 2006, 152:305-311.
26. Bateman A: The SIS domain: a phosphosugar-binding domain. Trends Biochem Sci 1999, 24:94-95.

27. Tong HH, Blue LE, James MA, De Maria TF: Evaluation of virulence of a Streptococcus pneumoniae neuraminidase-deficient mutant in nasopharyngeal colonization and development of otitis media in the chinchilla model. Infect Immun 2000, 68:921-924.

28. Orihuela CJ, Gao G, Francis KP, Yu J, Tuomanen El: Tissue-specific contributions of pneumococcal virulence factors to pathogenesis. J Infect Dis 2004, 190:1661-1669.

29. King SJ, Hippe KR, Weiser JN: Deglycosilation of human glycoconjugates by the sequential activities of exoglycosidases expressed by Streptococcus pneumoniae. Mol Microbiol 2006, 59:961-974

30. Burnaugh AM, Frantz LJ, King SJ: Growth of Streptococcus pneumoniae on human glycoconjugates is dependent upon the sequential activity of bacterial exoglycosidases. J Bacterio/ 2008, 190:221-230.

31. Hoskins J, Alborn WE, Arnold J, Blaszczak LC, Burgett S, Dehoff BS, et al: Genome of the bacterium Streptococcus pneumoniae strain R6. J Bacteriol 2001, 183:5709-5717.

32. Byers $\mathrm{HL}$, Homer KA, Beighton D: Utilisation of sialic acid by viridans streptococci. J Dent Res 1996, 75:1564-1571.

33. Vollmer W: Structural variation in the glycan strands of bacterial peptidoglycan. FEMS Microbiol Rev 2008, 32:287-306

34. Deutscher J, Francke C, Pot B, Postma PW: How phosphotransferase system-related protein phosphorylation regulates carbohydrate metabolism in bacteria. Microbiol Mol Biol Rev 2006, 70:939-1031.

35. Poncet S, Milohanic E, Maze A, Nait Abdallah J, Ake F, Larribe M, et al: Correlations between carbon metabolism and virulence in bacteria. Contrib Microbiol 2009, 16:88-102.

36. Iyer R, Baliga NS, Camilli A: Catabolite control protein (CcpA) contributes to virulence and regulation of sugar metabolism in Streptococcus pneumoniae. J Bacteriol 2005, 187:8340-8349.

37. van Opijnen T, Bodi KL, Camilli A: Tn-seq: high-throughput parallel sequencing for fitness and genetic interaction studies in microorganisms. Nat Methods 2009, 6:767-772.

38. Carvalho SM, Kloosterman TG, Kuipers OP, Neves AR: CcpA ensures optimal metabolic fitness of Streptococcus pneumoniae. PLoS One 2011, 6:e26707.

39. Novichkov PS, Laikova ON, Novichkova ES, Gelfand MS, Arkin AP, Dubchak I, et al: RegPrecise: a database of curated genomic inferences of transcriptional regulatory interactions in prokaryotes. Nucleic Acids Res 2009, 38:D111-D118.

40. Pearce BJ, lannelli F, Pozzi G: Construction of new unencapsulated (rough) strains of Streptococcus pneumoniae. Res Microbiol 2002, 153:243-247.

41. Pozzi G, Musmanno RA, Lievens PMJ, Oggioni MR, Plevani P, Manganelli R: Methods and parameters for genetic transformation of Streptococcus sanguis Challis. Res Microbiol 1990, 141:659-670.

42. Pozzi G, Musmanno RA, Renzoni EA, Oggioni MR, Cusi MG: Host-vector system for integration of recombinant DNA into chromosomes of transformable and nontransformable streptococci. J Bacteriol 1988, 170:1969-1972.

43. lannelli F, Pozzi G: Method for introducing specific and unmarked mutations into the chromosome of Streptococcus pneumoniae. Mol Biotechnol 2004, 26:81-86.

44. Chiavolini D, Memmi G, Maggi T, lannelli F, Pozzi G, Oggioni MR: The three extra-cellular zinc metalloproteinases of Streptococcus pneumoniae have a different impact on virulence in mice. BMC Microbio/ 2003, 3:14.

45. Carver T, Beriman M, Tivey A, Patel C, Böhme U, Barrell BG, et al: Artemis and $A C T$ : viewing, annotating and comparing sequences stored in a relational database. Bioinformatics 2008, 24:2672-2676.

46. Vickerman MM, lobst S, Jesionowski AM, Gill SR: Genome-wide transcriptional changes in Streptococcus gordonii in response to competence signaling peptide. J Bacterio/ 2007, 189:7799-7807.

47. Denapaite $D$, Bruckner R, Reichmann P, Henrich B, Maurer P, Schahle $Y$, et al: The genome of Streptococcus mitis B6 - what is a commensal? PLoS One 2010, 5:e9426.

48. Reichmann P, Nuhn M, Denapaite D, Bruckner R, Henrich B, Maurer P, et al: Genome of Streptococcus oralis strain Uo5. J Bacteriol 2011, 193:2888-2889.

49. Xu P, Alves JM, Kitten T, Brown A, Chen Z, Ozaki LS, et al: Genome of the opportunistic pathogen Streptococcus sanguis. J Bacteriol 2007, 189:3166-3175. 
50. Oggioni MR, lannelli F, Ricci S, Chiavolini D, Parigi R, Trappetti C, et al: Antibacterial activity of a competence-stimulating peptide in experimental sepsis caused by Streptococcus pneumoniae. Antimicrob Agents Chemother 2004, 48:4725-4732.

51. Livak KJ, Schmittgen TD: Analysis of relative gene expression data using real-time quantitative PCR and the 2(-Delta Delta C(T)) Method. Methods 2001, 25:402-408.

doi:10.1186/1471-2180-12-200

Cite this article as: Gualdi et al:: Regulation of neuraminidase expression in Streptococcus pneumoniae. BMC Microbiology 2012 12:200.

\section{Submit your next manuscript to BioMed Central and take full advantage of:}

- Convenient online submission

- Thorough peer review

- No space constraints or color figure charges

- Immediate publication on acceptance

- Inclusion in PubMed, CAS, Scopus and Google Scholar

- Research which is freely available for redistribution 